\title{
JAMES, WITTGENSTEIN E 0 PRAGMATISMO: APROXIMAÇÕES E DISTANCIAMENTOS
}

\author{
Filicio Mulinari ${ }^{1}$ \\ Universidade Federal de São Paulo (UNIFESP) \\ (D) https://orcid.org/0000-0002-6999-6956
}

\begin{abstract}
RESUMO:
Além de apontar os tópicos nos quais Wittgenstein mantém relação direta com o pensamento de James - ainda que de forma crítica -, almeja-se aqui demonstrar que os pensadores possuem pontos de convergência relevantes e, ainda, compartilham de compromissos filosóficos comuns, como a crítica ao antifundacionalismo, a prioridade da prática sobre a subjetividade e intelecto, o holismo no que tange a significação dos termos, dentre outros. Mais que isso, almeja-se também pontuar aqui em quais pontos a crítica de Wittgenstein a James realmente faz jus ao pensamento do autor americano. No mais, busca-se ainda o esclarecimento da noção de pragmatismo entendido por Wittgenstein, principalmente por sua possível relação teórica com James, a fim de responder sobre a possibilidade de se enquadrar ou não a filosofia de Wittgenstein sob a ótica pragmática. Assim sendo, torna-se necessário agora a realização de uma análise mais pormenorizada dos objetivos acima mencionados, bem como uma clarificação da problemática em torno deles.
\end{abstract}

PALAVRAS-CHAVE: Pragmatismo; Linguagem; James; Wittgenstein.

\section{JAMES, WITTGENSTEIN AND PRAGMATISM: SIMILARITIES AND DIFFERENCES}

\begin{abstract}
:
Besides pointing out the topics that Wittgenstein maintains a direct relationship with the thought of James - although critically - we aim here demonstrate that thinkers have important points of convergence and also share common philosophical commitments as critical the antifoundationalism, holism regarding the meaning of terms, among others. More than that, also aim to score points here in which Wittgenstein's
\end{abstract}

\footnotetext{
${ }^{1}$ Doutorando em filosofia pela Universidade Federal de São Paulo (UNIFESP).
} 
criticism of James truly lives up to the thought of American author. In most, it seeks further clarification of the notion of pragmatism understood by Wittgenstein, mainly for its possible theoretical relationship with James in order to answer about the possibility of falling or not Wittgenstein's philosophy under the pragmatic perspective. Therefore, it is necessary now to carry out a more detailed analysis of the aforementioned objectives, as well as a clarification of the issues around them.

KEYWORDS: Pragmatism; Language; James; Wittgenstein.

\section{Introdução}

Se não quisermos RESOLVER problemas filosóficos - por que não desistimos de nos ocupar com eles. Pois resolvê-los significa modificar o seu ponto de vista, o antigo modo de pensar. Se não o quiseres fazer, deves considerar os problemas como insolúveis. (WITTGENSTEIN, LWPPII, MS 174, § 24).

Apesar de negligenciado por muito tempo pela tradição analítica da filosofia, o pensamento de William James vem recebendo nos últimos anos uma análise mais dedicada e cuidadosa por parte dos filósofos dessa corrente. A atenção dada por Hillary Putnam à questão é um exemplo disso. ${ }^{2}$ Putnam aproxima James da tradição analítica ao associar o pensamento do autor ao pensamento de Wittgenstein, principalmente de seus escritos tardios, que envolvem as Investigações Filosóficas, os escritos sobre psicologia e o Da Certeza. ${ }^{3}$ Sobre essa relação entre os pensadores, Putnam afirma que:

Allow that William James might have had something 'new' to say - something new to $u s$, not just new to his own time - or, at

\footnotetext{
${ }^{2}$ Sobre o tratamento da questão, vide as obras de Putnam The Many faces of Realism (1987), Realism with a Human Face (1989) e Pragmatism (1995).

3 É importante observar uma questão existente em torno dos escritos tardios de Wittgenstein, sobretudo, os escritos de psicologia produzidos entre os anos de 1946 e 1949. Conforme assinala Schulte (1995, p. 1), são os escritos de psicologia de Wittgenstein um trabalho filosófico à parte, ou eles são parte de uma obra maior do filósofo? Dado que apenas o Tractatus Logico-Philosophicus e o Some Remarks of Logical Forms foram publicados com o filósofo em vida, a tentativa de dar uma resposta definitiva a tal questão se torna tanto quanto problemática. Não obstante, assinala-se de antemão que aqui, na presente pesquisa, se tomará partido da convicção de que os escritos sobre filosofia da psicologia de Wittgenstein são escritos com objetivos que, apesar de interligados a obras anteriores, são distintos de outros trabalhos, o que por sua vez concederia certa "autonomia" aos mesmos. Sobre isso, Marques (2007, p. 8-9) afirma categoricamente: "De facto, especialistas com a autoridade de G. H. Von Wright e Joachim Schulte, entre outros, consideram que as observações do último Wittgenstein sobre filosofia da psicologia representam realmente algo novo, uma reconfiguração de temas e conceitos, ainda que nunca se ponha em causa a total continuidade da metodologia e do estilo de pensamento".
} 
least, might have had a program for philosophy that is, in part, the right program, even if it has not been properly worked out yet...; if we allow that Husserl and Wittgenstein and Austin may have shared something of the same program, even if they too, in their different ways, failed to state it properly; then there is still something new, something unfinished and important to say about reality and truth. And that is what I believe (PUTNAM, 1987, p 17).

De fato, tal como pode ser entendido pela afirmação de Putnam, Wittgenstein pode ter compartilhado em sua filosofia, sob certo aspecto, algumas ideias de James. Não só isso, cabe ressaltar que ainda enquanto era aluno de Cambridge, em 1912, o filósofo austríaco teve acesso à obra Variedades da Experiência Religiosa (1902), de James. Sobre o livro, Wittgenstein chegou afirmar a Russell: "sempre que tenho tempo agora leio Variedades da Experiência Religiosa de James. Este livro tem me feito muito bem" (Wright, 1974). Cabe ainda ressaltar que após seu retorno à filosofia na década de 1930, a obra Princípios da Psicologia (1890) de James foi o único livro de filosofia visível nas estantes de Wittgenstein (Passmore, 1966, p. 434).

Além dos fatos mencionados acima, outros pontos ajudam a confirmar relação próxima que o filósofo vienense possuía com o pensamento de James. Por exemplo, algumas pessoas são mencionadas na primeira versão de sua obra póstuma Investigações Filosóficas (1953) e, dentre elas, estão Beethoven, Schubert, Göethe, dentre outros. No entanto, James é mencionado diretamente em quatro passagens da obra - apenas Agostinho, com cinco citações, é mais mencionado que o pensador americano. Não obstante, deve-se ter em mente que James não está somente presente nesses quatro momentos em que é citado diretamente, mas em várias outras passagens também é possível perceber uma referência a seus escritos no debate, ainda que de modo implícito. Sobre isso, cabe ressaltar a passagem de A. C. Jackson, um dos discípulos diretos de Wittgenstein, que diz:

Wittgenstein very frequently referred to James in his lectures, even making on one occasion - to everybody's astonishment a precise reference to a page number! (JACKSON apud PASSMORE, 1966, p. 434).

Apesar do nítido interesse, ainda que críticos, de Wittgenstein nos escritos de James, ressalta-se que ainda são poucos os trabalhos que fundamentam e aprofundam essa relação. ${ }^{4}$ Em tese, podem ser postos dois

\footnotetext{
${ }^{4}$ Certamente o trabalho do professor Goodman é uma valiosa excessão à essa regra. Em seu livro "James and Wittgenstein"(2002), Goodman relaciona o pensamento de ambos os filósofos, fazendo sobretudo questão de ressaltar seus pontos de convergência, principalmente no que tange ao pragmatismo.
} 
motivos particulares para a falta de pesquisas nessa área. Primeiramente, devido à divulgação tardia dos escritos de Wittgenstein que tratam questões relativas aquilo que o filósofo pontuou como filosofia da psicologia - são nesses escritos, juntamente com o Da Certeza, que Wittgenstein mantém maior relação, com os escritos de James.

Um segundo ponto importante para a escassez de pesquisas que abordem a relação existente entre James e Wittgenstein diz respeito às suas respectivas correntes filosóficas. Uma vez que ambos pensadores são tipicamente enquadrados em tradições distintas da filosofia contemporânea, a análise correlacionada dos autores torna-se mais complexa. Isso se dá porque, normalmente, comentadores e estudiosos de Wittgenstein tendem a não dar a devida atenção aos escritos de James - ou tendem a dar apenas atenção às partes explicitadas nas críticas de Wittgenstein. Por outro lado, aqueles que estudam James frequentemente possuem pouco conhecimento sobre Wittgenstein. Tais fatos justificam, em parte, a escassa atenção dada a tal questão na comunidade acadêmica durante certo tempo.

A fim de demonstrar a carência de trabalhos que abordem a relação de ambos autores, nota-se que alguns dos mais renomados comentadores das Investigações Filosóficas de Wittgenstein simplesmente ignoram a referência do pensador a James na obra. Esse é o caso, por exemplo, de David Pears (1987), David Stern (1995) e Colin McGinn (1984). Outros comentadores, como Robert Fogelin (1987) e Malcolm Budd (1993), até percebem a influência de James na obra, mas contentam-se em analisar essa influência dando atenção praticamente exclusiva às críticas proferidas por Wittgenstein a James, renegando possíveis influências que Wittgenstein poderia ter obtido por meio da leitura da obra do filósofo americano, bem como pontos de convergência teórica entre os autores.

Além dos pontos acima mencionados, há ainda outro importante questionamento em torno da relação de Wittgenstein com James: o questionamento do filósofo austríaco sobre o pragmatismo. De fato, Wittgenstein realiza tal questionamento em dois escritos específicos, a saber, no Da Certeza e na segunda parte das Investigações Filosóficas, também publicado como Observações sobre a Filosofia da Psicologia. Nas obras, Wittgenstein questiona se sua posição teórica poderia ser, em certo sentido, tomada como uma 'posição pragmatista'. Nota-se:

Mas você não é um pragmatista? Não. Pois não estou dizendo que uma sentença é verdadeira se ela é útil.

A serventia, isto é, o uso, dá à sentença seu sentido particular, o jogo de linguagem o dá a ela (RPPI, § 266).

Sobre o pragmatismo, tema presente em Wittgenstein - muito frequentemente presente nos escritos de forma oculta -, a pergunta a ser respondida aqui é: qual uso Wittgenstein faz do termo 'pragmatista'? Qual sentido ele atribui a tal termo para negá-lo? Essa pergunta conduz ao retorno 
às obras de Wittgenstein, às questões que o filósofo coloca e leva ao engajamento realizado pelo filósofo ao longo de sua vida em conjuntura com os escritos de William James.

Nesse sentido, além de apontar os tópicos nos quais Wittgenstein mantém relação direta com o pensamento de James - ainda que de forma crítica -, almeja-se aqui demonstrar que os pensadores possuem pontos de convergência relevantes e, ainda, compartilham de compromissos filosóficos comuns, como a crítica ao antifundacionalismo, a prioridade da prática sobre a subjetividade e intelecto, o holismo no que tange a significação dos termos, dentre outros. Mais que isso, almeja-se também pontuar aqui em quais pontos a crítica de Wittgenstein a James realmente faz jus ao pensamento do autor americano. No mais, busca-se ainda o esclarecimento da noção de pragmatismo entendido por Wittgenstein, principalmente por sua possível relação teórica com James, a fim de responder sobre a possibilidade de se enquadrar ou não a filosofia de Wittgenstein sob a ótica pragmática. Assim sendo, torna-se necessário agora a realização de uma análise mais pormenorizada dos objetivos acima mencionados, bem como uma clarificação da problemática em torno deles.

\section{Divergências: as críticas de Wittgenstein a James}

Em seu livro Princípios da Psicologia, pontualmente no capítulo 9, denominado de A corrente do Pensamento [The Stream of Thought], James descreve alguns traços básicos da noção de interior. Um dos objetivos de James com essa análise é corrigir a visão tradicional de consciência e interioridade contidas nas teorias de Locke e Hume. Segundo James, de acordo com a concepção tradicional presente nas obras dos referidos filósofos, há a ideia de que o indivíduo sempre é consciente de objetos e sensações e que, ainda, todo conteúdo mental é constituído a partir de "imagens [...] de coisas perfeitamente definidas" (James, 1890, p. 246). Entretanto, para James essa visão seria equivocada sobre os processos mentais. Para o pensador americano, além de estarmos conscientes dos objetos e de suas qualidades sensíveis, também estaríamos conscientes das relações entre tais objetos por meio daquilo que ele denominou como sentimentos de relação, que seriam uma peça chave na compreensão do pensamento. Sobre isso, tem-se:

Devemos dizer um sentimento de $e$, um sentimento de $s e$, um sentimento de mas e um sentimento de por, praticamente tão prontamente como dizemos um sentimento de azul ou um sentimento de frio. Todavia, não dizemos: tão inveterado se tornou o nosso hábito de reconhecer a existência das partes substantivas somente que a linguagem quase se recusa a emprestar-se a qualquer outro uso (JAMES, 1890, p. 238). 
Vale lembrar que, além dos sentimentos de relação, existe ainda aquilo que o pensador denominou de sentimentos de tendência:

Jamais o leitor se perguntou que tipo de fato mental é a sua intenção de dizer uma coisa antes que a dissesse? É uma intenção inteiramente definida, distinta de todas as outras intenções, um estado absolutamente distinto de consciência, portanto; e todavia o quanto dela consiste de imagens sensoriais definidas, seja de palavras ou de coisas? Dificilmente alguma coisa! [...]Ela tem, portanto, uma natureza própria do tipo mais positivo, e todavia o que podemos dizer sobre ela sem fazer uso de palavras que pertencem aos posteriores fatos mentais que a substituem? A intenção de dizer-tal-e-tal é o único nome que ela pode receber (JAMES, 1890, p. 245).

Segundo James, haveria diferentes experiências conscientes relativas à pretensão de enunciar cada sentença específica, i.e., a intenção de enunciar a sentença $X$ envolveria uma experiência distinta da intenção de enunciar a sentença Y. Para exemplificar esse ponto, James dá o exemplo de quando uma palavra está "na ponta da língua":

Suponha-se que tentamos recordar um nome esquecido. O estado da nossa consciência é peculiar. Há um hiato ali dentro: mas não é nenhum mero hiato. É um hiato que é intensamente ativo. Um tipo de espectro do nome está nele, acenando-nos em uma dada direção, fazendo-nos, em momentos, comichar com o sentido da nossa proximidade e deixando-nos então reemergir sem o termo ensejado. Se nomes errados são propostos a nós, esse hiato singularmente definido age imediatamente de modo a negá-los. Eles não se encaixam em seu molde. E o hiato de uma palavra não faz sentir como o hiato de outra, todos vazios de conteúdo como ambos poderiam parecer necessários ser; quando descritos como hiatos. Quando em vão tento recordar o nome de Spalding, a minha consciência está de muito afastada do que acontece quando tento em vão recordar o nome de Bowles (JAMES, 1890, p.243).

Wittgenstein está em concordância com James no que diz respeito à rejeição da ideia de que a consciência seria composta de imagens e sensações postas em sucessão, tal como pontua a tradição empirista: para ambos, o conteúdo da experiência mental vai muito além disso. No entanto, segundo Wittgenstein, James cairia no erro de incluir como características da experiência consciente processos mentais que não seriam sequer experiências. Este seria o caso, por exemplo, dos sentimentos de relação e de tendência. Nesse viés, podemos fazer três questionamentos distintos à filosofia de James em concordância com as ideias do filósofo austríaco.

Primeiramente, pode-se questionar a ideia de James de que teríamos diferenciadas experiências - ou sentimentos de relação - para as noções de 
$e, s e$, mas, etc. Factualmente, ainda que existissem sentimentos relativos ao uso da palavra "se", e.g., para Wittgenstein seria equivocado pensar que esse sentimento seria sempre o mesmo cada fez que tal palavra fosse usada:

Estamos certos de que há um único sentimento-de-se, e não talvez diversos? Tentamos dizer a palavra em uma grande variedade de contextos? Por exemplo, quando ele porta a ênfase principal da sentença, e quando a palavra seguinte o faz (IF-II, vi-181-182).

Um segundo ponto tencionado por Wittgenstein acerca do tema proposto por James diz respeito ao uso da palavra. Noutros termos, se um indivíduo compreende o termo "se", tal fato mantém relação com o uso que se faz do termo, não de um suposto modo como a palavra é "sentida" pelo indivíduo.

Suponha que achamos um homem que, falando sobre como as palavras se fizeram sentir para ele, contou-nos que "se" e "mas" fizeram sentir o mesmo. - Podemos não acreditar nele? Poderíamos achar isso estranho. "Ele não joga o nosso jogo em absoluto", alguém poderia dizer. Ou mesmo: "Esse é um tipo diferente de ser humano". Se ele fizesse uso das palavras "se" e "mas" como fazemos uso, não pensaríamos que ele as entendeu como nós entendemos? (IF-II, vi-182)

Como se percebe, para Wittgenstein é o uso que merece uma atenção maior para o tratamento da questão, e não supostos fenômenos intrínsecos à consciência do sujeito. Sob essa perspectiva, podemos caracterizar um terceiro momento da crítica de Wittgenstein: quando um sujeito possui uma sensação de um tipo particular em conexão ao pronunciar o termo "se", tal sensação não é algo que condiciona o uso de tal termo, i.e., não é em todos os casos que possuímos tal sensação ao usar o termo "se". Ao contrário, tal sensação só se mostra ao passo que damos a devida atenção e enfocamos nos sentimentos que temos ao fazer o uso de tal termo: o "jogo de linguagem" nesse caso seria o de "sentir o significado de uma palavra". Contudo, o fato de termos sentimentos nesse momento não nos assegura que, no uso ordinário costumeiro, esses sentimentos sempre estão presentes.

Se atenção cuidadosa me mostra que, quando estou jogando esse jogo, experiencio a palavra ora dessa maneira, ora daquela maneira - isso também não me mostra que na corrente de discurso eu com frequência não experiencio em absoluto a palavra? (IF-II, xi-215-216).

Certamente as críticas de Wittgenstein a James são pontuais e relevantes. Porém, é de grande importância ressaltar que, em alguns aspectos, é difícil imaginar que o próprio James defenderia algumas das posições que são alvos da crítica de Wittgenstein. Como exemplo, tem-se: 
James dificilmente argumentaria que um "sentimento-de-se" é uma condição necessária e/ou eficiente para o uso significativo da palavra "se", como afirma o primeiro ponto. Além disso, dificilmente James iria se opor à ideia de Wittgenstein de que não haveria nenhum sentimento único do "sentimento-de-se", tal como afirma a segunda objeção. Como o próprio James rejeita, é difícil imaginar que a mesma grama nos dá, sempre, o mesmo sentimento de verde, bem como dificilmente o céu nos daria sempre o mesmo sentimento de azul, etc. (vide JAMES , 1983, p. 225-227). Da mesma forma, é de se esperar que ele rejeite a ideia de que a mesma relação nos dê, efetivamente, sempre o mesmo sentimento de relação. Porém, no que diz respeito à terceira crítica proposta por Wittgenstein, é necessário uma melhor análise.

O terceiro ponto da crítica de Wittgenstein diz respeito à ideia de que experienciamos os sentimentos de relação somente em situações nas quais estamos de fato procurando por tais sentimentos. Isto, de fato, é oposto à teoria de James. Para James, os sentimentos de relação seriam elementos sempre presentes em nossa percepção consciente. Dessa forma, caso Wittgenstein esteja correto em sua posição, isso significaria que James apresenta de maneira equivocada o caráter de nossa vida consciente, sobretudo por fazer análises e fundamentar experiências que de fato não possuímos.

Segundo Wittgenstein, James equivoca-se ao representar a natureza dos fenômenos mentais ao relacioná-los a "sentimentos de tendência". Para Wittgenstein, James trata erroneamente a intenção de dizer alguma coisa como uma experiência. Nesse sentido, James afirma que:

[...] mesmo antes que tenhamos aberto nossas bocas para falar, o pensamento inteiro está presente à nossa mente na forma de uma intenção de enunciar aquela sentença (JAMES, 1890, p.269).

A passagem acima faz com que Wittgenstein afirme que, para James, "o pensamento já está completo no começo da sentença" (RPPI, 173). Sob a ótica do filósofo, a concepção de James forçaria a visão de que, ao enunciar uma sentença, algo vai à mente do indivíduo antes mesmo de enunciá-la.

Wittgenstein aponta que o que sustenta essa ideia de que haveria "algo" na mente antes mesmo do sujeito proferi-la seria a capacidade do falante de, ao ser interrompido, continuar o enunciado que ele mesmo tinha iniciado. Ora, uma vez que a sentença já passou pela consciência antes mesmo do indivíduo enunciá-la, seria fácil entender o motivo da facilidade do mesmo indivíduo continuar a sentença após a interrupção. Contudo, Wittgenstein apresenta algumas objeções a essa visão.

Primeiramente, a ideia da existência anterior ao pronunciamento do enunciado cria uma imagem ilusória do que realmente ocorre nesses casos. Nesse sentido, tem-se que quando uma pessoa se lembra do que estava 
dizendo, logo após ser interrompido, normalmente isso não ocorre ao se recordar um estado de consciência na qual se estava no momento de dizer tal enunciado. Seria errôneo, ainda, pensar que é possível recordar fielmente de um processo mental que se passava no momento exato no que se iria proferir algo. Nesses casos, o indivíduo simplesmente lembraria do que estava proferindo sem a necessidade de recordar qualquer experiência.

\begin{abstract}
Antes, você foi interrompido; você ainda sabe o que queria dizer [sagenwollen]? Se agora sei, e o digo - significa isso que antes já o pensara e somente não o dissera? Não. A menos que você tome a certeza a qual eu continuo a frase interrompido como critério para o fato de que o pensamento já estava pronto naquela ocasião. - Mas já havia certamente tudo o que era possível - para auxiliar a continuação da frase, na situação e nos meus pensamentos. (IF $\S 633$ )
\end{abstract}

Dessa forma, não haveria nenhum fundamento para a afirmação de James de que antes mesmo de ser proferido, o enunciado já estaria presente em seu sentido completo na mente do sujeito. Além disso, de acordo com as ideias de Wittgenstein, James cometeria um erro grave ao tratar a intenção de dizer algo como um tipo de experiência.

Para James, o sujeito possuiria um "estado de consciência distinto" antes de pronunciar uma sentença que, de algum modo, revelaria a sentença antes mesmo dela ser pronunciada. Nas palavras de Wittgenstein, seria como se "tudo o que [eu sigo dizendo] já estava ali desde o começo e estava contido na experiência inicial" (LWPPI. 843). Para o filósofo austríaco, seria errôneo observar nesse tipo de experiência algo como um "germe lógico" (LWPPI, 843) das sentenças. Esse modo de enxergar a significação das palavras seria, para Wittgenstein, errôneo. A explicitação dessa crítica constitui um dos é um dos pontos auges da relação entre os teóricos no que diz respeito às divergências existentes entre o pensamento de ambos.

\title{
Convergência: método pragmático e terapias filosóficas
}

Apesar das explicitadas divergências entre James e Wittgenstein, ainda assim aproximações podem ser feitas e, nesse sentido, uma das mais relevantes e influentes diz respeito ao método pragmático e o modo como Wittgenstein lida com os problemas filosóficos em sua última fase. Em seus últimos anos de vida, Wittgenstein fez duas considerações sobre uma possível relação entre sua filosofia e o pragmatismo. Cabe ressaltar que tais considerações foram feitas em contextos distintos e a explicitação dessa conjuntura merece melhor análise.

A primeira referência de Wittgenstein ao pragmatismo aparece em sua obra Da Certeza: "So I am trying to say something that sounds like pragmatism. Here I am being thwarted by a kind o Weltanschauung" (OC, 422). É importante delimitar aqui o que Wittgenstein significa por 
pragmatismo e, ainda, quais características de sua posição "soam" como pragmatismo. Afinal, a posição wittgensteiniana realmente soa como pragmatista para o próprio filósofo ou, de forma distinta, meramente aparenta ser um pragmatismo, mas sem realmente ser? Para responder essa questão é necessário fazer uma incursão sobre o conhecimento de Wittgenstein sobre pragmatismo e, após isso, passar para a discussão do autor sobre esse tema no Da Certeza.

Primeiramente, cabe analisar o conjunto de parágrafos datados de um dia antes da única aparição do termo "pragmatismo" na obra $D a$ Certeza.

Our knowledge forms an enormous system. And only within this system has a particular bit the value we give it (OC, 410).

If I say "we assume that the earth has existed for many years past" (or something similar), then of course it sounds strage that we should assume such a thing. But in the entire system of our language-games it belongs to the foundations. The assumpition, one might say, forms the basis of action, and therefore, naturally, of thought. (OC, 411).

Os parágrafos acima exibem duas características da filosofia de Wittgenstein que podem ser compartilhadas com o pragmatismo de James, a saber, o sentido de que nem todas as proposições - inclusive as de crença jogam a mesma regra e, ainda, a inter-relação entre ação e pensamento. Por meio delas, nota-se a complexa descrição de Wittgenstein sobre regras e proposições que envolvem atos de crença, como a crença de que "a Terra existiu há muitos anos atrás". Este tipo de proposição pode aparecer em uma crença pertencente a um "cético radical", e.g., mas não aparece em afirmações "não filosóficas", i.e., quando se está fora do jogo de linguagem filosófico. Cada crença para Wittgenstein forma uma base de ação e de pensamento. Nesse sentido, se a Terra é colocada em questão, como se pode pensar que eu mesmo vivi com minha mãe, na minha cidade natal, há alguns anos atrás? É nesse sentido que se afirma que o modo de crença é uma base para ação e, depois, para o pensamento. Aqui, o ponto chave para a compreensão se dá por meio da visão holística das ideias de Wittgenstein.

Much of the work of On Certainty lies in an attempt to explain the nature of this system, and the book contains a series of forceful and beautiful metaphors expressing the idea that some propositions are under contention or exploration, while others are outside the domain of inquiry. Those outside the domain of inquiry are, as it were, already tacitly "decided" upon - not by any individual or group individuals, but by the human culture living within the framework they provide. Wittgenstein speaks of our "frame of reference", versus the facts we discover within the frame $(\mathrm{OC}, 83)$; of the route traveled by inquiry versus the places inquiry does not go (OC, 88); of the "inherited background" versus the truths we discover against this 
background (OC, 94); of convictions lying on an "unused siding" (OC, 210) versus those on the main line; and of the "hinges" of all else that we do (GOODMAN, 2002, p. 21).

O parágrafo 411 do Da Certeza lança luz sobre essa natureza holística do processo de significação, sugerindo que a ação é dada juntamente com o "pensamento". Vale lembrar que o Da Certeza trabalha a luz das ideias das Investigações Filosóficas, que afirmam que a linguagem toma forma nos jogos de linguagem, com suas complexas formas de vida que incluem construir, contar piadas, ordenar, etc. (IF, §23). Wittgenstein frequentemente aborda o fundamento da relação entre ação e pensamento como um conjunto de proposições referidas como "imagem de mundo" [world-picture]. Entretanto, também de forma frequente, o filósofo diz: o "fim" de questões de justificação não deve ser tratado como uma proposição simplesmente, mas como um conjunto de atos, i.e., como uma "forma de vida". No que diz respeito a esse aspecto holístico da significação, é razoável indicar que há convergência de ideias entre James e Wittgenstein.

James and Wittgenstein both find authority in the lived world, whether thought of as "the stream of thought" or as "language games", "pure experience" or "forms of life". Obviously the reliance on ordinary language is more fully developed in Wittgenstein, but it is nevertheless completely in accord with a main tendency in James, [...] both early and late help us to see (GOODMAN, 2002, p. 161).

Além do parágrafo 422 do Da Certeza, que como se mostrou trata das considerações de Wittgenstein sobre o pragmatismo e um possível tratamento holístico ao processo de significação dos termos, há ainda outra parte onde Wittgenstein considera sua relação com o pragmatismo: as notas datadas de 1947, publicadas postumamente sob o nome de Observações sobre a Filosofia da Psicologia, vol. I. Nessa obra, o filósofo austríaco centra sua atenção na relação entre termos psicológicos e significado. $\mathrm{Na}$ obra, ele questiona: "But you aren't a pragmatist? No. For I am not saying that a proposition is true if it is useful" (RPPI, 266).

Antes de analisar a resposta de negativa de Wittgenstein à questão, é essencial compreender os fundamentos que levaram o filósofo a essa afirmação, analisando as partes que circundam a negação. Para isso, cabe analisar um parágrafo anterior a referida citação:

E quando, porém, a religião ensina que a alma pode subsistir após a corrupção do corpo? Eu entendo o que ela ensina? Sem dúvida, eu entendo - posso imaginar uma porção de coisas aqui. (Até pintaram quadros sobre essas coisas. E por que um desses quadros deveria ser apenas uma versão inacabada do pensamento proferido? Por que ele não deve realizar o mesmo serviço que nossas sentenças?) E é o serviço que importa. 
(RPPI, 265)

Mas você não é um pragmatista? Não. Pois não estou dizendo que uma sentença é verdadeira se ela é útil.

A serventia, isto é, o uso, dá à sentença seu sentido particular, o jogo de linguagem o dá a ela.

E já que uma regra é frequentemente dada de forma que ela se prova útil, e as sentenças matemáticas estão essencialmente relacionadas a regras, o que se reflete nas verdades matemáticas é a utilidade. (RPPI, 266)

A justaposição da questão do pragmatismo com a série de questionamentos sobre religião sugere, mais uma vez, a relevância da obra de Variedades da Experiência Religiosa, de James, para o pensamento de Wittgenstein. Ressalta-se que a obra Variedades da Experiência Religiosa contém uma variedade de pontos relativos ao pragmatismo, ou, noutros termos, uma vasta gama de doutrinas e princípios pragmatistas em seu tratamento das distintas experiências religiosas. Desse modo, se o pragmatismo está presente nas Observações sobre a Filosofia da Psicologia (e consequentemente nas Investigações Filosóficas), essa obra de James lança luz sobre o entendimento de quão vasto e profundo pode ser o pensamento de Wittgenstein em torno do significado de "pragmatismo".

Embora o escrito Pragmatismo seja a obra mais emblemática de James referente ao tema, fato interessante é visto ao se analisar a definição de pragmatismo dada por James na obra Variedades da Experiência Religiosa. ${ }^{5}$ No capítulo intitulado "Filosofia" [Philosophy], ele introduz sua noção por meio do conceito de pragmatismo definido por Peirce:

To attain perfect clearness in our thoughts of an object, we need then only consider what sensations, immediate or remote, we are conceivably to expect from it, and what conduct we must prepare in case the object should be true. Our conception of these practical consequences is for us the whole of our conception of the object, so far as that conception has positive significance at all (JAMES, 1987, p. 399).

Como se nota, James não está, na citação cima, tratando de um pragmatismo enquanto uma teoria da verdade, mas sim de termos como "conceituação" ou "significação". Outra passagem pode confirmar a ideia de que James, na passagem, faz referência sobre uma possível teoria do significado.

\footnotetext{
${ }^{5}$ Segundo Lovejoy (1963, p. 1-29), James - em sua obra Pragmatism - distingue quatro significados distintos para o termo "pragmatismo": a) o pragmatismo como teoria da verdade; b) como teoria do conhecimento; c) como método e, por fim, d) como teoria do significado ou significante. Este último carrega importância particular para o referido escrito de Wittgenstein.
} 
To develop a thought's meaning we need therefore only determine what conduct it is fitted to produce; that conduct is for us its sole significance; and the tangible fact at the root of all our thought-distinctions is that there is no one of them so fine as to consist in anything but a possible difference of practice (JAMES, 1987, p.399)

$\mathrm{Na}$ citação, James propõe que o significado dos termos religiosos é abstraído da variedade de práticas e experiência das quais se descreve e, nesse sentido, não há a necessidade de uma definição primeira para, após isso, conhecer o que essas práticas e experiências de fato são e significam. Sobre esses apontamentos, Goodman esclarece:

For our purposes in discussing Remarks, we should notice how clearly James here separates the question of meaning from that truth. The significance of religious terms, unlike the truth of claims made using them, is precisely not a hypothesis; it is established by the utility of believing it. He does not embrace here the crude pragmatic theory of his detractors, including Wittgenstein: that the true is the useful. Rather, he holds a view much like the Wittgensteinian view that provokes Wittgenstein's question about his own pragmatism - that the "service" to wich we put religious pictures, or religious teachings, provides or constitutes something we "understand" (GOODMAN, 2002, p. 154).

Embora seja possível concentrar a atenção no pragmatismo como uma teoria do significado e traçar relações entre esse ponto e as ideias de Wittgenstein, outro fator também torna ainda a aproximação possível entre os dois pensadores: o método pragmático adotado por James e a ideia de filosofia enquanto conjunto de diferente terapias para Wittgenstein.

James pensa o pragmatismo como um método para resolver principalmente controvérsias metafísicas intermináveis. Wittgenstein, na mesma esteira de pensamento, visa diferentes métodos em filosofia assim como "diferentes terapias" (IF, 133). Ambos compactuam a ideia de que a filosofia trata de seus próprios excessos e patologias: para Wittgenstein, o 'enfentiçamento por meio da linguagem' (IF, 109); para James “inveterate habits dear to professional philosophers" (Pragmatism, p. 31). Embora Wittgenstein não pareça estar com essas semelhanças em mente em sua afirmação negativa sobre ser um pragmatista, uma aproximação com James parece, no mínimo ser possível ou razoável. 


\section{Referências bibliográficas}

BUDD, Malcolm. Wittgenstein's Philosophy of Psychology. $2^{\mathrm{a}}$ Edição. Padstow, Cornwall, Great Britain: Routledge London and New York. 1993. CAVELL, Stanley. The Claim of Reason: Wittgenstein, Skepticism, Morality, and Tragedy. Oxford: Oxford University Press, 1979.

FOGELIN, Robert J. Wittgenstein, 2nd ed. New York and London: Routledge, 1987

JAMES, William. The Varieties of Religious Experience. New York: Longmas, Green and Co., 1917.

.Writings 1902-1910 Literary Classics of the United Stares, Inc., New York, 1987.

The Principles of Psychology. Cambridge, MA: Harvard University Press, 1983.

LOVEJOY, Arthur. The Thirteen Pragmatisms. Baltimore: The Johns Hopkins University Press, 1963.

McGINN, Colin. Wittgenstein on Meaning .Oxford: Basil Blackwell, 1984. PUTNAM, Hillary. The many faces of realism. La Salle, IL: Open Court, 1987.

1995.

Pragmatism. An open question. Oxford: Blackwell publishers,

PEARS, D. The False Prison. 2 vols. Oxford: Clarendon Press, 1987-8

SCHULTE, Joachim. Experience and Expression: Wittgenstein's Philosophy of Psychology. Oxford: Clarendon Press, 1995.

STERN, David G. Wittgenstein on Mind and Language. Oxford and New York: Oxford University Press, 1995.

WITTGENSTEIN, Ludwig. On certainty. G.E.M. Ansombe and G.H. von Wright (eds.). Oxford: Blackwell, 1969.

- Tractatus Logico-Philosophicus. D Pears and McGuinnes (Tr),

London: Routledge \& Kegan Paul, 1974.

. Philosophical Remarks. Rush Rhees (Ed.) Oxford: Basil Blackwell, 1975.

Remarks on the Philosophy of Psychology. Vol. I. G.E.M.

Anscombe and G.H. von Wright (eds). Oxford: Basil Blackwell, 1980. Remarks on the Philosophy of Psychology. Vol. II. Luckhardt, C. G, and Aue, M (eds). Oxford: Basil Blackwell, 1980.

Last Writings on the Philosophy of Psychology, Vol I. ed. G.H. von

Wright and Heikki Nyman, trans. C.G. Luckhardt and Maximiliam Aue. Chicago: University of Chicago Press, 1982

. Philosophical Investigations. The German Text, with a Revised

English Translation. 3 ed. G.E.M. Anscombe (Ed. e Tr.), Oxford: Blackwell Publishing, 2001. 